\title{
Association between microalbuminuria and metabolic syndrome in patients with rheumatoid arthritis Samia Abdelmonem ${ }^{\mathrm{a}}$, Refaat Eltanawy ${ }^{\mathrm{a}}$, Yaser Ismail ${ }^{\mathrm{b}}$, Eman Baraka ${ }^{\mathrm{c}}$, Asmaa Shoshan ${ }^{d}$
}

\author{
aprofessor of Rheumatology, Rehabilitation and \\ Physical Medicine, ${ }^{b}$ Professor of Clinical \\ Pathology, ${ }^{\mathrm{C}}$ Assistant Professor of \\ Rheumatology, Rehabilitation and Physical \\ Medicine, ${ }^{\mathrm{d}}$ Assistant Lecturerof Rheumatology, \\ Rehabilitation and Physical Medicine, Faculty \\ of Mednicie, Benha University, Egypt \\ Correspondence to Dr. Asmaa Shoshan, MSc, \\ Assistant Lecturer of Rheumatology, \\ Rehabilitation and Physical Medicine, Faculty \\ of Mednicie, Benha University, Egypt. \\ e-mail: asmashoshan@gmail.com \\ Received 23 July 2017 \\ Accepted 19 October 2017
}

Egyptian Rheumatology \& Rehabilitation 2018, 45:87-93

\begin{abstract}
Context
Rheumatoid arthritis (RA) is an autoimmune, symmetrical polyarticular disease characterized by chronic inflammation of the synovial joints. Microalbuminuria (MA) occurs as leakage of small amounts of albumin into the urine. Metabolic syndrome (MetS) describes the risk factors for cardiovascular diseases such as dyslipidemia, obesity, hypertension, and diabetes.

Aim

The aim of this study was to detect the prevalence of MA in patients with RA and study its correlation with disease activity and severity. Our aim extends to identify the association of MA with MetS in RA.

Patients and methods

This study was carried out on 30 adult RA patients, $30 \%$ of them were men and $70 \%$ were women (mean \pm SD: $42.27 \pm 10.99$ years). Their mean disease duration was $12.8 \pm 7.06$ years. A total of 20 apparently healthy adults, age-matched and sexmatched served as a control group. All the patients were subjected to full history taking, full clinical examination, laboratory investigations, and assessment of disease activity using the disease activity score for 28 joints score. Urinary microalbumin level was measured in all participants in early morning samples by the immunoturbidometry method. MetS was assessed in all participants according to Grundy's criteria.

Results

The frequency of MetS was highly statistically significant in patients with RA compared with the control group. The RA patients' group had highly significantly elevated mean values of urinary microalbumin and urinary albumin to creatinine ratio compared with the control group.

\section{Conclusion}

MA and MetS are frequent in RA, particularly in those with long-standing disease. Early detection of albuminuria allows early intervention with the goal of reducing inflammation development in RA, cardiovascular risk. MetS is frequent in RA patients with MA.
\end{abstract}

\section{Keywords:}

cardiovascular disease, metabolic syndrome, microalbuminuria, rheumatoid arthritis

Egypt Rheumatol Rehabil 45:87-93

(C) 2018 Egyptian Society for Rheumatology and Rehabilitation

1110-161X

\section{Introduction}

Rheumatoid arthritis (RA) is an autoimmune, symmetrical polyarticular disease which is characterized by chronic inflammation of the synovial joints affecting primarily the diarthroidal joints. The pathogenic characteristic features of RA includes synovial hyperplasia and inflammation accompanied by cartilage loss and joint destruction, which result in extremely painful, impaired functional status that produces substantial morbidity as well as an increase in mortality [1].

In a properly functioning body, albumin is not normally present in urine because it is retained in the blood stream by the kidney. Microalbuminuria (MA) occurs as a leakage of small amounts of albumin into the urine, when there is an abnormal high permeability for albumin in the renal glomerulus of the kidney. MA is not a small albumin molecule found in the urine, but it is defined as 30-299 $\mathrm{mg}$ of albumin being lost in urine per day undetectable by the dipstick method. In proteinuria, the level of protein in urine is more than or equal to $300 \mathrm{mg}$ a day [2].

In RA, glomerular proteinuria is considered as a complication of advanced disease caused by either direct effects of the disease on the kidney or actions of nephrotoxic drugs or both. However, subclinical renal dysfunction is not uncommon in RA and many patients with incipient nephropathy are not detected by routine laboratory assays for urine total protein. An elevated urinary albumin index and estimation of MA is useful for

This is an open access journal, and articles are distributed under the terms of the Creative Commons Attribution-NonCommercial-ShareAlike 4.0 License, which allows others to remix, tweak, and build upon the work non-commercially, as long as appropriate credit is given and the new creations are licensed under the identical terms. 
the finding of subclinical glomerular injury in patients with RA [3]. Renal dysfunction might be detected earlier by the appearance of MA [4].

RA associates with cardiovascular disease (CVD); the strength of this association may be comparable to that observed with diabetes mellitus. The inflammatory background of RA patients would subject them to a higher prevalence of MA. Furthermore, MA could theoretically serve as a useful tool for cardiovascular $(\mathrm{CV})$ risk stratification [5].

The metabolic syndrome (MetS) describes a constellation of major risk factors for CVD such as atherogenic dyslipidemia, obesity, hypertension, and diabetes. These associated risk factors have been previously called syndrome $X$, or the insulin resistance syndrome. The National Cholesterol Education Program's Adult Treatment Panel III (NCEP-ATPIII) and in 2005, the International Diabetes Federation, defined the MetS as the metabolic complications of obesity with the inclusion of the clinical characteristics of abdominal obesity (increased waist circumference), atherogenic dyslipidemia, hypertension, and raised fasting plasma glucose concentrations [6].

In this work, we aimed to detect the prevalence of MA in patients with RA and study its correlation with disease activity and severity. Our aim extends to identify the association of MA with the MetS which describes the risk factors linked to CVD disease in RA.

\section{Patients and methods}

\section{Patients}

A total of 30 patients fulfilling the 2010 American College of Rheumatology/European League against Rheumatism classification criteria for RA were included in the study. Their ages ranged between 21 and 68 years. Totally, 20 apparently healthy participants were included as controls. Patients and controls were recruited from the attendants of the inpatients' unit and outpatients' clinic of the Rheumatology, Rehabilitation and Physical Medicine Department, Benha University Hospitals. Patients with evidence of previous renal diseases or proteinuria and patients using nephrotoxic drugs were excluded from the study.

A written informed consent was obtained from all the patients and controls according to the principles of the ethics committee of Benha Faculty of Medicine.

All patients underwent a thorough baseline evaluation including a detailed review of their medical history, physical examination, and disease activity score for 28 joints (DAS28) calculation. Venous blood samples were collected in the fasting state on the same day of clinical evaluation. Laboratory investigations were performed including full and differential blood count, erythrocyte sedimentation rate (Westergren method), a full biochemical panel, C-reactive protein (CRP), and fasting lipids and glucose. Early morning, midstream urine samples were collected from all the patients and urinary albumin concentration was determined by the immunoturbidometric assay. Urinary creatinine was measured by an endpoint Jaffe reaction. For standardization of urine concentration, urinary albumin was expressed as the ratio of urinary albumin to urinary creatinine $(\mu \mathrm{g} / \mathrm{mg})$.

Each patient's current medication was recorded with special interest in potentially nephrotoxic drugs. Traditional CVD risk factors recorded for each patient included: (a) blood pressure, (b) fasting lipid profile, (c) BMI calculation $\left(\mathrm{kg} / \mathrm{m}^{2}\right)$, and (d) waist circumference measurement $(\mathrm{cm})$. The MetS was defined by the updated Grundy's criteria 2005 .

Participants were classified as having MA if the level of urinary microalbumin was greater than $15 \mathrm{mg} / 1$ or albumin: creatinine ratio was more than $30 \mu \mathrm{g} / \mathrm{mg}$.

\section{Statistical analysis}

Statistical analysis was performed using the statistical package for social sciences software, version 16 (SPSS; SPSS Inc., Chicago, Illinois, USA). Clinical and laboratory variables were presented as mean $\pm \mathrm{SD}$ for continuous data and as number (\%) for qualitative ones. Student's $t$-test was used for comparisons of continuous data between groups, whereas $\chi^{2}$-test was used for categorical data. Level of evidence less than 0.05 was considered statistically significant. Correlations were evaluated using Spearman's rank correlation coefficient $(r)$.

\section{Results}

Thirty RA patients [nine (30\%) men and 21 (70\%) women] aged from 21 to 68 years (mean \pm SD: $42.27 \pm$ 10.99 years) together with 20 [four (20\%) men and 16 $(80 \%)$ women] aged from 18 to 65 years $(m e a n \pm S D$ : $39.7 \pm 13$ years) were enrolled in the study. The mean duration of symptoms among RA patients was $12.8 \pm$ 7.06 years with a range of $1-22$ years.

Demographic, clinical, and laboratory characteristics among RA patients and control group are presented in Table 1 . The RA patients' group had highly significantly 
Table 1 Demographic, clinical, and laboratory characteristics among rheumatoid arthritis patients and control group

\begin{tabular}{|c|c|c|c|}
\hline Variables & RA patients $(N=30)($ mean $\pm S D)$ & Control group $(N=20)($ mean $\pm S D)$ & $P$ value \\
\hline Waist circumference $(\mathrm{cm})$ & $1.03 \pm 0.13$ & $1.06 \pm 0.16$ & 0.558 \\
\hline $\mathrm{SBP}(\mathrm{mmHg})$ & $131.3 \pm 24.9$ & $112 \pm 17.35$ & $0.004^{*}$ \\
\hline $\mathrm{DBP}(\mathrm{mmHg})$ & $83.8 \pm 13.4$ & $73.5 \pm 8.75$ & $0.004^{*}$ \\
\hline FBS $(\mathrm{mg} / \mathrm{dl})$ & $181.4 \pm 132.04$ & $90.3 \pm 12.34$ & $0.004^{*}$ \\
\hline TGs (mg/dl) & $178.6 \pm 98.8$ & $69.7 \pm 15.14$ & $<0.001^{\star *}$ \\
\hline $\mathrm{HDL}(\mathrm{mg} / \mathrm{dl})$ & $43.4 \pm 17.5$ & $52.0 \pm 8.98$ & $0.048^{*}$ \\
\hline ESR first hour (mm/h) & $47.17 \pm 37$ & $10 \pm 4.4$ & $0.042^{*}$ \\
\hline C-reactive protein (mg/l) & $46.5 \pm 31.11$ & $6.8 \pm 9.9$ & $<0.001^{* *}$ \\
\hline Blood urea $(\mathrm{mg} / \mathrm{dl})$ & $35.13 \pm 0.178$ & $29 \pm 7.78$ & $0.002^{*}$ \\
\hline Serum creatinine (mg/dl) & $0.92 \pm 0.233$ & $0.96 \pm 0.157$ & 0.541 \\
\hline Urinary microalbumin (mg/l) & $63.9 \pm 2.57$ & $21.95 \pm 13.88$ & $<0.001^{* *}$ \\
\hline $\mathrm{A} / \mathrm{CR}(\mu \mathrm{g} / \mathrm{mg})$ & $46.6 \pm 35.28$ & $14.9 \pm 11.45$ & $<0.001^{\star *}$ \\
\hline MetS $[n(\%)]$ & $18(60)$ & $2(10)$ & $<0.001^{\star *}$ \\
\hline
\end{tabular}

$\mathrm{A} / \mathrm{CR}$, urinary albumin to creatinine ratio; DBP, diastolic blood pressure; ESR, erythrocyte sedimentation rate; FBS, fasting blood sugar; HDL, high-density lipoprotein; MetS, metabolic syndrome; RA, rheumatoid arthritis; SBP, systolic blood pressure; TGs, triglyceride. $P>0.05=$ insignificant. ${ }^{\star} P<0.05=$ significant. ${ }^{* \star} P<0.001=$ highly significant.

Table 2 Comparisons of the clinical status and current medication in the rheumatoid arthritis patients' group according to the frequency of the metabolic syndrome

\begin{tabular}{|c|c|c|c|c|c|}
\hline Variables & Metabolic syndrome $[n(\%)]$ & No metabolic syndrome $[n(\%)]$ & Total number & $\chi^{2}$-test & $P$ value \\
\hline \multicolumn{6}{|l|}{ Clinical manifestations } \\
\hline \multicolumn{6}{|l|}{ Articular } \\
\hline Arthritis $(n=30)$ & $18(60)$ & $12(40)$ & 30 & 0.003 & 0.54 \\
\hline \multicolumn{6}{|l|}{ Extra-articular } \\
\hline SC nodules $(n=1)$ & $1(100)$ & $0(0.0)$ & 1 & 3.25 & $0.041^{*}$ \\
\hline Pleurisy $(n=5)$ & $3(60)$ & $2(40)$ & 5 & 1.00 & 0.68 \\
\hline Conjunctivitis $(n=3)$ & $3(100)$ & $0(0.0)$ & 3 & 2.24 & $0.025^{*}$ \\
\hline \multicolumn{6}{|l|}{ Disease activity grades 28 score } \\
\hline Mild $(n=1)$ & $1(100)$ & $0(0.0)$ & 1 & 0.702 & 0.704 \\
\hline Moderate $(n=10)$ & $6(60)$ & $4(40)$ & 10 & & \\
\hline Severe $(n=19)$ & $11(58)$ & $8(42)$ & 19 & & \\
\hline \multicolumn{6}{|l|}{ Current medications } \\
\hline Antimalarial $(n=9)$ & $5(56)$ & $4(44)$ & 9 & 0.11 & 0.75 \\
\hline Corticosteroids (25) & $15(60)$ & $10(40)$ & 25 & 1.1 & $0.04^{*}$ \\
\hline $\operatorname{MTX}(n=19)$ & 7 (37) & $12(63)$ & 19 & 11.6 & $0.002^{*}$ \\
\hline Salazopyrine $(n=11)$ & $4(36)$ & $7(64)$ & 11 & 4.04 & $0.044^{*}$ \\
\hline Leflunomide $(n=17)$ & $7(41)$ & $10(59)$ & 17 & 5.8 & $0.016^{*}$ \\
\hline Microalbuminuric & $13(87)$ & $2(13.3)$ & 15 & 8.889 & $0.003^{*}$ \\
\hline Disease duration (mean $\pm S D$ ) & $10.6 \pm 5.1$ & $6.2 \pm 0.85$ & & 3.16 & $0.004^{*}$ \\
\hline
\end{tabular}

MTX, methotrexate; SC, subcutaneous. $P>0.05=$ insignificant. ${ }^{*} P<0.05=$ significant.

elevated mean values of systolic blood pressure (SBP), diastolic blood pressure (DBP), fasting blood sugar (FBS), triglycerides, high-density lipoprotein (HDL), erythrocyte sedimentation rate (ESR) first hour, CRP, and blood urea compared with the control group. The RA patients' group had highly significantly elevated mean values of urinary microalbumin and urinary albumin to creatinine ratio $(\mathrm{A} / \mathrm{CR})$ compared with the control group. The frequency of the MetS according to Grundy's criteria was $60 \%$ in the RA patients' group. This frequency was highly statistically significant compared with the control group (10\%).

Table 2 shows comparisons of the clinical status and current medication in the RA patients' group according to the frequency of MetS. Patients with arthritis and pleurisy had an increased prevalence of MetS that did not reach statistical significance, while there were statistically significant differences for RA patients with subcutaneous nodules $(P=0.041)$ and conjunctivitis $(P=0.025)$ regarding the frequency of the MetS.

Regarding the RA disease activity, the MetS was associated with different disease grades with no statistical significance between them. Statistically increased significant differences in the frequency of the MetS was observed in patients treated with corticosteroids $(P=0.04)$, whereas the frequency was also increased in patients on antimalarial drugs with no statistical difference $(P=0.75)$. The frequency of 
patients without MetS statistically increased in patients treated with methotrexate (MTX) $(P=0.002)$, salazopyrine $(P=0.044)$, and leflunomide $(P=0.016)$.

The MetS was prevalent in 13/15 (87\%) of RA patients with MA, whereas five (33\%) normoalbuminuric RA patients had the MetS. A statistically significant difference was observed among these groups $(P<0.05)$. On the other hand, $13 / 18(72 \%)$ of RA patients with MetS had MA. RA patients with the MA had a statistically significantly higher mean disease duration $(P=0.004)$ than patients without the MetS.

Table 3 shows comparisons between some clinical and laboratory parameters in RA patients according to the presence of MA.

Although RA patients with MA had increased mean values of disease duration, BMI, waist circumference, FBS, triglycerides, HDL, DAS28, and Larsen's score, they had no statistically significant differences compared with normoalbuminuric patients.

Table 4 shows Spearman's correlation coefficients between urinary microalbumin levels and some clinical and laboratory variables in RA patients' group. There were highly statistically significant positive correlations $(P<0.001)$ of urinary microalbumin levels with disease duration, duration of morning stiffness, number of tender joints, number of swollen joints, ESR first hour, CRP, Larsen's score, DAS28, and A/CR.

\section{Discussion}

MA is one of the important biomarkers involved in various disorders related to $\mathrm{RA}$. The prevalence of MA in $\mathrm{RA}$ is higher than that reported in the general population. It appears predominantly to associate with hypertension and not with inflammation or therapy and may be a marker for RA patients with the MetS [7].

The epidemiology of MA shows a close association with systemic endothelial dysfunction, vascular disease, and also implicates glomerular endothelial dysfunction [8].

A predominant effect of sex hormones on the pathogenesis of RA is evidenced by the disproportionate number of women with this disease [9]. This was confirmed in our study where nine (30\%) patients were men and 21 (70\%) patients were women.

In our study, the RA patients' group had a statistically significantly higher frequent MA than the control group (50 vs. $10 \% ; P<0.001)$. Meanwhile, these patients had a highly significantly elevated mean values of urinary $\mathrm{A} / \mathrm{CR}$ ratio $(P<0.001)$ compared with the control group.

In a study by Pederson et al. [10], MA prevalence in nondiabetic, nonhypertensive participants, was significantly higher in RA patients compared with the controls (27.7 vs. $7.8 \%$ ). However, that cohort included many patients on gold or penicillamine which are rarely used nowadays and have been associated with MA in RA. Another large-scale study by Sihvonen et al. [8] focusing on the role of renal disease as a predictor of survival, which did not explore potential associations with CVD risk factors, showed a similar prevalence of MA among 604 (5.63\%) RA patients and 457 (5.91\%) age-matched and sexmatched controls. In a study of 39 nondiabetic, nonhypertensive RA patients made by Mpofu et al. [11], the prevalence of MA was $7.7 \%$, a percentage comparable to that of the general population. A study made by Dimitrios et al. [12] showed that an overall

Table 3 Comparisons between some clinical and laboratory parameters in rheumatoid arthritis patients according to presence of microalbuminuria

\begin{tabular}{|c|c|c|c|c|}
\hline Variables & $\begin{array}{l}\text { Normoalbuminuric patients }(N=15) \\
(\text { mean } \pm S D)\end{array}$ & $\begin{array}{l}\text { Microalbuminuric patients }(N=15) \\
(\text { mean } \pm S D)\end{array}$ & $t$-Test & $P$ value \\
\hline Disease duration (years) & $11.4 \pm 7.4$ & $14.2 \pm 6.7$ & 1.06 & 0.297 \\
\hline $\mathrm{BMI}\left(\mathrm{kg} / \mathrm{m}^{2}\right)$ & $35.42 \pm 6.45$ & $36.32 \pm 6.51$ & 0.38 & 0.71 \\
\hline Waist circumference $(\mathrm{cm})$ & $1.046 \pm 0.142$ & $1.02 \pm 0.11$ & 0.45 & 0.659 \\
\hline SBP $(\mathrm{mmHg})$ & $128.67 \pm 10.6$ & $128 \pm 6.76$ & 0.2 & 0.839 \\
\hline $\mathrm{DBP}(\mathrm{mmHg})$ & $82 \pm 7.75$ & $80.67 \pm 7.98$ & 0.46 & 0.646 \\
\hline FBS (mg/dl) & $111.2 \pm 37.67$ & $146.13 \pm 79.37$ & 0.5 & 0.135 \\
\hline TGs (mg/dl) & $164.67 \pm 37.2$ & $178.8 \pm 51.56$ & 0.86 & 0.397 \\
\hline $\mathrm{HDL}(\mathrm{mg} / \mathrm{dl})$ & $58.4 \pm 24.98$ & $61.6 \pm 25.94$ & 0.34 & 0.733 \\
\hline DAS28 & $6.09 \pm 1.79$ & $6.16 \pm 1.22$ & 0.132 & 0.896 \\
\hline Larsen's score & $2.26 \pm 1.22$ & $3.1 \pm 1.25$ & 1.92 & 0.065 \\
\hline
\end{tabular}

DAS, disease activity score; DBP, diastolic blood pressure; FBS, fasting blood sugar; HDL, high-density lipoprotein; SBP, systolic blood pressure; TG, triglyceride. 
Table 4 Spearman's correlation coefficients between urinary microalbumin levels and some clinical and laboratory variables in rheumatoid arthritis patients' group

\begin{tabular}{|c|c|c|}
\hline \multirow[t]{2}{*}{ Variables } & \multicolumn{2}{|c|}{$\begin{array}{l}\text { Level of urinary } \\
\text { microalbumin }\end{array}$} \\
\hline & $r$ & $P$ value \\
\hline Age & 0.306 & 0.1 \\
\hline Disease duration & 0.917 & $<0.001^{* *}$ \\
\hline Morning stiffness duration & 0.914 & $<0.001^{* *}$ \\
\hline Number of tender joints & 0.673 & $<0.001^{\star *}$ \\
\hline Number of swollen joints & 0.687 & $<0.001^{\star *}$ \\
\hline Weight & 0.137 & 0.469 \\
\hline Height & 0.210 & 0.266 \\
\hline Waist circumference & 0.258 & 0.169 \\
\hline BMI & 0.041 & 0.828 \\
\hline SBP & -0.228 & 0.226 \\
\hline DBP & -0.188 & 0.320 \\
\hline Hemoglobin \% & 0.108 & 0.570 \\
\hline WBCs & -0.156 & 0.411 \\
\hline PLT & 0.034 & 0.858 \\
\hline ESR first hour & 0.616 & $<0.001^{* *}$ \\
\hline C-reactive protein & 0.892 & $<0.001^{* *}$ \\
\hline Rheumatoid factor & -0.224 & 0.233 \\
\hline FBS & 0.107 & 0.575 \\
\hline Blood urea & 0.332 & 0.073 \\
\hline Serum creatinine & 0.306 & 0.1 \\
\hline TGs & 0.228 & 0.225 \\
\hline $\mathrm{HDL}$ & -0.064 & 0.736 \\
\hline Protein in 24 urine & 0.210 & 0.266 \\
\hline Larsen's score & 0.859 & $<0.001^{\star *}$ \\
\hline DAS28 & 0.866 & $<0.001^{\star *}$ \\
\hline $\mathrm{A} / \mathrm{CR}$ & 0.590 & $<0.001^{\star *}$ \\
\hline
\end{tabular}

$\mathrm{A} / \mathrm{CR}$, urinary albumin to creatinine ratio; DAS28, disease activity score; DBP, diastolic blood pressure; ESR, erythrocyte sedimentation rate; FBS, fasting blood sugar; HDL, high-density lipoprotein; PLT, platelet count; SBP, systolic blood pressure; TG, triglyceride; WBC, white blood cells. Protein in 24 hour urine collection better to delete this item. $P>0.05=$ insignificant. ${ }^{\star \star} P<0.001=$ highly significant.

prevalence of MA of $11.9 \%$ in patients with RA (including those with diabetes or hypertension), a percentage higher to that reported for the general population $(7.2 \%)$ in the largest scale study performed in more than 40000 participants aged 28-75 years made by Hillege et al. [13]. These results were inconsistent from ours. This discrepancy from our study may be explained by the method of microalbumin detection or number of participants.

A study made by Jensen et al. [14] in the detection of MA, the nosographic sensitivity and specificity, and the diagnostic specificity were 58,97 , and $66 \%$ for urinary microalbumin, and 73,97 , and $73 \%$ for urinary $\mathrm{A} / \mathrm{CR}$, respectively. They concluded that the measurement of the $\mathrm{A} / \mathrm{CR}$ concentration ratio is a specific and quite sensitive alternative to the measurement of the urinary albumin excretion rate in timed collections, when screening for MA.
In this study, MA correlated with the duration of RA, where patients with MA had a longer disease duration with no statistical difference between both groups $(P<0.297)$. Pederson et al. [10] found that patients with MA had a significantly higher mean duration of RA than the group with normal urinary level of microalbumin $(P<0.05)$. A highly statistically significant correlation of morning stiffnessduration $(P<0.001), \mathrm{CRP}(P<0.001)$, and ESR $(P<0.001)$ with urinary microalbumin level was found in our RA patients. This supported the findings of Pederson et al. [10]

We also confirmed the results of Dawnay et al. [15] and Pederson et al. [10] who found an association between urinary excretion of albumin and CRP. The possible correlation between urinary excretion of microalbumin, disease activity, and duration of RA disease can be explained in two ways, either a long-standing RA disease tends to affect the kidney and the systemic vascular permeability more or patients that with a longstanding disease receive more nephrotoxic systemic treatment [7].

According to Grundy's criteria, our RA patients had a statistically significant higher frequency of MetS (60\%) compared with the control group (10\%). Several studies have demonstrated that inflammatory processes are involved in the pathogenesis of MetS. On the other hand, there is evidence that components of $\mathrm{CV}$ risk increase the inflammatory burden in RA [16]. The current results are consistent with the study done by Chung et al. [17] which showed that MetS was significantly more prevalent in American patients with long-standing RA (42\% WHO and NCEP/ ATPIII criteria) as well as in early RA patients (31 and $30 \%$ WHO and NCEPIII criteria, respectively) than in the controls (11 and 22\% WHO and NCEPIII criteria, respectively). Karvounaris et al. [18] have found a low prevalence of MetS (defined with NCEP/ATPIII criteria) in $40 \%$ of RA patients compared with their control population and documented a relationship between disease activity and the presence of MetS. On the contrary, Sahebari et al. [19] have found that MetS was to be more common in the normal population (45.2\%) than in the RA patient (30.8\%). This great diversity in the frequency of the MetS between our study and others can be attributed to the fact that the prevalence of the MetS in RA depends on the definition used [20]. At present, six definitions for MetS have been established. These definitions have similarities; however, they differ in some of the components as well as in their specified cut-offs and weights. Moreover, differences in 
ethnicity, geographic area, study design, population studied, differences in the baseline characteristics, and the lifestyle of the population studied may clear the disparity. For example, older patients may have more disabilities leading to a sedentary lifestyle compared with younger populations. It can affect lipid profile and blood glucose levels and, therefore, have an impact on the prevalence of MetS [21].

In our study, RA patients had a statistically significantly higher SBP $(P=0.004), \quad$ DBP $\quad(P=0.004), \quad$ FBS $(P=0.004)$, TG $(P<0.001)$, and HDL $(P=0.048)$ than the control group, however, there were no statistically significant difference between both groups regarding the mean value of waist circumference $(P=0.558)$. This agreed with Dessein et al. [21] who stated that traditional risk factors such as HTN, central obesity, and dyslipidemia occur more frequently among patients with RA. The clustering of risk factors associated with central obesity (elevated glucose, dyslipidemia, and elevated blood pressure), often referred to as the MetS, is associated with a $2-5$-fold increased risk for the development of type 2 diabetes and a 3-4-fold increased incidence of $\mathrm{CV}$ diseases [6]. This is not in conformity with Karvounaris et al. [18], who found that controls were more likely to have hypercholesterolemia (75 vs. $63 \% ; P=0.001$ ) and high HDL (64 vs. $54 \%$; $P=0.01)$. Moreover, Sahebari et al. [19] have found that SBP, DBP, serum levels of TG, LDL were significantly higher in healthy controls $(P<0.05)$, whereas FBS was higher in RA patients $(P<0.05)$. This difference could be explained by the large number of controls included in their study.

Our result differs from those found by Karvounaris et al. [18] and Dao et al. [22], who found a correlation between DAS28 and individual component of MetS namely SBP $(P=0.01)$ and a trend toward an inverse correlation with HDL $(P=0.056)$. Besides, they found that significantly more patients without MetS had a low DAS index less than 3.2 compared with patients with MetS (16.5 vs. $2.8 \%$; $P=0.003$ ). Sahebari et al. [19] have claimed that DAS28 ESR is only a singlepoint measure of disease activity, whereas for RA, this will vary over time. A single measure does not give a very effective estimation of disease activity overall, and this may be a reason for the lack of association between disease activity and presence of MetS. Therefore, using multiple markers could give a better estimation of disease activity and its association with MetS.

In the present study, a statistically increased significant difference in the frequency of MetS was observed in patients treated with corticosteroids $(P=0.04)$.
The frequency was also increased in patients on antimalarial drugs with no statistical difference $(P=0.75)$. The frequency of patients without MetS statistically increased in patients treated with MTX $(P=0.002)$, salazopyrine $(P=0.044)$, and leflunomide $(P=0.016)$. A study made by Toms et al. [24] have shown that MTX use, but not other disease modifying antirheumatic drugs or glucocorticoids, was associated with significantly reduced chance of having the MetS in RA ( $P=0.004)$. A study made by Dao et al. [22] have found that Vietnamese women with early RA already had a higher prevalence of MetS compared with healthy controls. Less MTX uses were independent predictors associated with the presence of MetS in women with early RA. These findings suggest that physicians should screen for MetS in women with early RA to control its components and therefore reduce their risk of CV diseases. Chakib et al. [23] have demonstrated that concerning medication use in RA there was a significant relationship between the presence of MetS and glucocorticoid use with high doses and with NSAIDs use. Several meta-analyses have shown that NSAIDs are associated with an increased risk of $\mathrm{CV}$ events with the exception of naproxen, but no significant results were found for the risk of developing MetS in RA. For glucocorticoids, the results were similar to those of Chung et al. [17] who reported a higher prevalence of MetS in RA patients taking glucocorticoids. In other studies, this association was not found. The role of glucocorticoids is complex. On the one hand, longterm use at high doses is associated with adverse lipid profiles, hyperglycemia, HTN, and central obesity that contribute to the development of MetS. The negative association between MTX use and the presence of MetS, suggesting its protective effect against the development of MetS, may be attributed to an antiinflammatory effect or a drug-specific effect (because it was not observed with any of the other disease modifying antirheumatic drugs or biologic agents).

The MetS was prevalent in 13/15 (86.6\%) of our RA patients with MA, whereas five (33.3\%) normoalbuminuric RA patients had the MetS. A statistically significant difference was observed among these groups regarding the frequency of MetS $(P<0.05)$. A cross-sectional analysis made by Cirillo et al. [25] on 1567 participants aged 45-64 years, without diabetes mellitus, and with fasting plasma glucose levels of less than $7.8 \mathrm{mmol} / 1$, blood pressure, plasma cholesterol levels, smoking, and BMI significantly related to urinary albumin excretion and prevalence of MA. In analyses with controls for multiple variables, they concluded that major $\mathrm{CV}$ 
risk factors, e.g. male sex, higher SBP, higher plasma cholesterol level, smoking, and higher BMI are independent correlates of MA in nondiabetic middle-aged adults.

The presence of MA can be regarded as an index of increased $\mathrm{CV}$ vulnerability and a signal for vigorous efforts at correction of known risk factors [8].

\section{Conclusion}

$\mathrm{MA}$ and MetS prevalence was frequent in RA and they mutually increased the occurrence of each other in a long-standing disease with higher disease activity. Detection of MA and/or MetS allows introduction of aggressive measures to reduce inflammation and subsequent $\mathrm{CV}$ risks. Such patients may require more aggressive screening and management approaches for CVD risk factors. Interventions are needed to modify the progression of MetS and/or MA at an early time point after the onset of the RA disease.

\section{Financial support and sponsorship \\ Nil.}

\section{Conflicts of interest}

There are no conflicts of interest.

\section{References}

1 Ramesh BN, Mahalakshmi AM. Present clinical approaches to rheumatoid arthritis: an ample review. Indo Am J Pharma Sci 2013; 3:6055-6065.

2 American Diabetes Association. Nephropathy in diabetes. J Diabetes Care 2004; 27(Suppl 1):79-83.

3 Bhatt G, Mathur DS, Saxena GN, Bhanadari S. Microalbuminuria in rheumatoid arthritis: a correlation with disease activity. J Assoc Physicians India 2002; 50:82

4 Lars MP, Henrik N, Birthe S, Henning B. Microalbuminuria in patients with rheumatoid arthritis. Ann Rheum Dis 1995; 54:189-192.

5 Stamatelopoulos KS, Kitas GD, Papamichael CM, Chryssohoou E, Kyrkou $\mathrm{K}$, Georgiopoulos $\mathrm{G}$, et al. Atherosclerosis in rheumatoid arthritis versus diabetes: a comparative study. Arterioscler Thromb Vasc Biol 2009; 29:1702-1708.

6 Grundy SM, Cleeman JI, Daniels SR, Donato KA, Eckel RH, Franklin BA, et al. Diagnosis and management of the metabolic syndrome. An American Heart Association/National Heart, Lung, and Blood Institute Scientific Statement. Circulation 2005; 112:e285-e290.

7 Nidavani RB, Mahalakshmi AM, Patil JS. Microalbuminuria as risk indicator in rheumatoid arthritis: an updated review. Int J Pharm Clin Res 2014; 6:138-145.
8 Sihvonen S, Korpela M, Mustonen J, Laippala P, Pastemack A. Renal disease as a predictor of increased mortality among patients with rheumatoid arthritis. Nephron Clin Pract 2004; 96:c107-c114.

9 Cynthia CS, Silvia R, George D, Sherine E, Gabriel SE, et al. Challenge of developing a cardiovascular risk calculator for patients with rheumatoid arthritis. PLoS One 2017; 12:e0175605.

10 Pederson LM, Nordin H, Svensson B, Bliddal H. Microalbuminuria in patients with rheumatoid arthritis. Ann Rheum Dis 1995; 54:189-192.

11 Mpofu S, Kaushik VV, Grundy G, Moots RJ. Microalbuminuria: it is a predictor of ischemic heart disease in rheumatoid arthritis. Rheumatology (Oxford) 2004; 43:537-538.

12 Dimitrios D, Vasileios F, Panoulas VF, loannis A. Microalbuminuria in rheumatoid arthritis in the post penicillamine/gold era. Clin Rheumato 2011; 30:477-484.

13 Hillege HL, Fidler V, Diercks GF, van Gilst WH, de Zeeuw D, van Veldhuisen DJ, et al. Urinary albumin excretion predicts cardiovascular and noncardiovascular mortality in general population. Circulation 2002 106:1777-1782.

14 Jensen JS, Clausen P, Borch K, Jensen G. Detecting microalbuminuria by urinary albumin/creatinine concentration ratio. Nephrol Dial Transplant 1997; 12:6-9.

15 Dawnay A, Wilson AG, Lamb E, Kirby JD, Cattell WR. Microalbuminuria in systemic sclerosis. Ann Rheum Dis 1992; 51:384-388.

16 Gremese E, Ferraccioli G. The metabolic syndrome: the crossroads between rheumatoid arthritis and cardiovascular risk. Autoimmun Rev 2011; 10:582-589.

17 Chung CP, Oeser A, Solus JF. Inflammation- associated insulin resistance: differential effects in rheumatoid arthritis and systemic lupus erythematosus define potential mechanisms. Arthritis Rheum 2008; 58:2105-2112.

18 Karvounaris SA, Sidiropoulos PI, Papadakis JA, Spanakis EK, Bertsias GK Kritikos HD, et al. Metabolic syndrome is common among middle-to-older aged Mediterranean patients with rheumatoid arthritis and correlates with disease activity: a retrospective, cross-sectional, controlled, study. Ann Rheum Dis 2007; 66:28-33.

19 Sahebari M, Goshayeshi L, Mirfeizi Z, Rezaieyazdi Z, Hatef MR, GhayourMobarhan M, et al. Investigation of the association between metabolic syndrome and disease activity in rheumatoid arthritis. ScientificWorldJournal 2011; 11:1195-1205.

20 Toms TE, Panoulas VF, Douglas KM, Griffiths HR. Lack of association between glucocorticoid use and presence of the metabolic syndrome with rheumatoid arthritis: a cross-sectional study. Arthritis Res Ther 2009; 10: R145.

21 Dessein $\mathrm{PH}$, Norton GR, Joffe BI, Abdool-Carrim AT, Woodiwiss AJ, Solomon A. Metabolic cardiovascular risk burden and atherosclerosis in African black and Caucasian women with rheumatoid arthritis: a crosssectional study. Clin Exp Rheumatol 2013; 31:53-61.

22 Dao HH, Do QT, Sakamoto J. Increased frequency of metabolic syndrome among Vietnamese women with early rheumatoid arthritis: a crosssectional study. Arthritis Res Ther 2010; 12:R218.

23 Chakib N, Fatima A, Samia M, Adil N, Latifa T, Taoufik H. Prevalence of metabolic syndrome in patients with rheumatoid arthritis in Morocco: a cross-sectional study. Clin Rheumatol 2014; 33:1549-1555.

24 Toms TE, Panoulas VF, Douglas KM, Griffiths HR, Kitas GD. Lack of association between glucocorticoid use and presence of the metabolic syndrome in patients with rheumatoid arthritis: a cross-sectional study. Arthritis Res Ther 2008; 10:R145.

25 Cirillo M, Senigalliesi L, Laurenzi M, Alfieri R, Stamler J, Stamler R, et al. Microalbuminuria in nondiabetic adults: relation of blood pressure, body mass index, plasma cholesterol levels, and smoking. Arch Intern Med 1998; 158:1933-1939. 